\title{
Ridotti livelli plasmatici di testosterone o diidrotestosterone, ma non di estradiolo, sono associati a sintomi di claudicatio intermittens in uomini anziani
}

\author{
Fabio Lanfranco • Stefano Allasia
}

Pubblicato online: 11 marzo 2014

(C) Springer International Publishing AG 2014

\section{Commento a: \\ Lower plasma testosterone or dihydrotestosterone, but not estradiol, is associated with symptoms of intermittent claudication in older men. \\ B.B. Yeap, H. Alfonso, S.A. Chubb, D.J. Handelsman, G.J. Hankey, J. Golledge, L. Flicker and P.E. Norman. Clinical Endocrinology (2013) 79, 725-732}

Le malattie cardiovascolari e, in particolare, la malattia coronarica, sono tuttora al primo posto fra le cause di decesso tra i maschi. L'invecchiamento determina una progressiva riduzione della secrezione di testosterone nei maschi. L'effetto negativo della carenza di testosterone è stato dimostrato da alcuni recenti studi epidemiologici, che hanno evidenziato un' associazione tra bassi livelli di testosterone e aumento della mortalità totale e cardiovascolare. L'associazione tra livelli plasmatici di testosterone (T) e dei suoi metaboliti diidrotestosterone (DHT) ed estradiolo (E2) e la presenza di arteriopatia periferica sintomatica rimane, tuttavia, ancora poco chiara.

In questo studio retrospettivo sono stati coinvolti 3689 maschi di età compresa tra 70 e 89 anni, residenti a Perth (Australia); l'obiettivo dello studio è stato quello di valutare l'associazione tra T, DHT, E2 e insorgenza di arteriopatia obliterante periferica sintomatica.

I soggetti esaminati venivano considerati positivi per claudicatio intermittens solamente se il dolore alle gambe compariva durante il cammino, regrediva entro 10 minuti con il riposo e non interessava le articolazioni (Edinburgh Claudication Questionnaire).

F. Lanfranco $(\varangle) \cdot$ S. Allasia

Divisione di Endocrinologia, Diabetologia e Metabolismo,

Dipartimento di Scienze Mediche, Università di Torino, Torino,

Italia

e-mail: fabio.lanfranco@unito.it
Tra i pazienti, 268 presentavano dolore agli arti inferiori con le caratteristiche della claudicatio intermittens, 986 dolore aspecifico di natura non ischemica, mentre 2435 erano asintomatici. Dopo aver confrontato i dati ottenuti per età, fumo di sigaretta, BMI, rapporto vita/fianchi, dislipidemia, ipertensione, diabete mellito, creatinina e prevalenza di CVD, è risultato che elevati livelli di testosterone correlavano con un minor rischio di sviluppare claudicatio intermittens $(\mathrm{OR}=0,80$, IC $0,69-0,94, p=0,006$; quartili $\mathrm{Q} 4 / \mathrm{Q} 1 \mathrm{OR}=0,54$, IC $0,36-0,81)$. Anche livelli elevati di DHT risultavano protettivi (OR $=0,86$, IC $0,73-1,00$; quartili Q4/Q1 OR =0,64, IC 0,43-0,95), mentre ridotti livelli di E2 non aumentavano il rischio di sviluppare vasculopatia periferica sintomatica.

Lo studio ha dunque evidenziato come livelli ridotti di testosterone o del suo principale metabolita diidrotestosterone si associno a una maggiore probabilità di manifestare claudicatio intermittens nella popolazione maschile anziana, indipendentemente dalla presenza di ulteriori fattori di rischio cardiovascolare. Dallo studio emerge, invece, che i livelli di estrogeni circolanti non hanno alcun effetto sulla comparsa di vasculopatia periferica sintomatica.

Gli androgeni esercitano, infatti, una potente azione antiaterogena nel maschio sia mediante un'azione diretta sui vasi sanguigni, sia attraverso un meccanismo indiretto legato alla loro capacità di modulare la sensibilità periferica all'azione insulinica.

Ulteriori studi saranno necessari per valutare con maggiore chiarezza il ruolo protettivo degli androgeni nei confronti della comparsa di arteriopatia periferica sintomatica nei maschi anziani, allo scopo di migliorare la stratificazione del rischio e definire i trattamenti più opportuni. 